\title{
Biophysical Approach to Knee Osteoarthritis Pain and Disability
}

\author{
Alberto Foletti, Paolo Baron
}

\begin{abstract}
Knee osteoarthritis is a common disease leading to pain and disability in an increasing number of people. Since osteoarthritis is a progressive and degenerative skeletal disease, any therapy aimed to deal with it has to face from one side with the management of pain and recovery from disability; and from the other side with the slowing of osteoarthritis progression itself. Biophysical therapies have been shown to be efficacious in the treatment of pain in comparison to a common non-steroidal anti inflammation drug as ibuprofen, and to placebo. Moreover, biophysical therapy showed its effectiveness in the management of low back pain and disability, even at different degrees of intensity. Biophysical therapies exert, very likely, their effects though a resonance phenomenon. The aim of this study was to assess the possible efficacy of a biophysical protocol for knee osteoarthritis. Fifty consecutive patients (age 52.7 \pm 13.8 ), with different levels of pain and disability participate into this study employing an electro medical device (Med Select) to perform the electromagnetic information transfer through aqueous system (Nomabit Base) protocol according to previous reports. Assessment was done at baseline, and after 3 months, by the Oxford knee pain and disability questionnaire. Patients were classified as having a severe (score 0-19), moderate (score 20-29), mild (score 30-39), or satisfactory (score 40-48) knee condition. A global reduction in the knee pain and disability score was observed after 3 months of a single biophysical intervention, in fact mean start level was $2.1 \pm 0.71$, while mean end level was $0.24 \pm 0.52$ ( $p<0.0001)$. We hope that biophysical treatments are considered for larger and longer trials in order to meet the requirements to be widespread as a possible integrative support to the number of people with knee osteoarthritis in order to both manage pain and disability, as well as slowing the gonarthrotic progression.
\end{abstract}

Index Terms - Biophysical therapy, bioelectronic medicine, knee osteoarthritis, electromagnetic information.

\section{INTRODUCTION}

Knee osteoarthritis is a common disease leading to pain and disability in an increasing number of people [1]. Since osteoarthritis is a progressive and degenerative skeletal disease [2], any therapy aimed to deal with it has to face from one side with the management of pain and recovery from disability; and from the other side with the slowing of osteoarthritis progression itself. Biophysical therapies have been shown to be efficacious in the treatment of pain in comparison to a common non-steroidal anti inflammation drug as ibuprofen, and to placebo [3]. Moreover, biophysical therapy showed its effectiveness in the management of low

Alberto Foletti, Clinical Biophysics International Research Group, Lugano, Switzerland, and Institute of Translational Pharmacology, National Research Council-CNR, Rome Italy

Paolo Baron, Clinical Biophysics International Research Group, Lugano, Switzerland, and General practitioner, Palmanova, Italy back pain and disability [4], even at different degrees of intensity [5]. Additionally, a biophysical therapy showed its effectiveness in the improvement of cervical spine mobility [6], and in the management of fluctuating asymmetry [7]. Biophysical therapies exert, very likely, their effects though a resonance phenomenon [8]. The number of patients suffering from joint's pain and disability is continuously increasing due to aging of population highlighting some unmet medical needs and raising some challenges for personalized treatments [9]. The aim of this study was to assess the possible efficacy of a biophysical protocol for knee osteoarthritis as a model for the management of osteoarthritis in general.

\section{MATERIAL AND METHODS}

\section{II.1. Study Design.}

This was an open-label prospective cohort study.

\section{II.2. Study Population.}

Patients with diagnosis of knee osteoarthritis following X-ray examination, and scoring from level 1 (mild) to level 3 (severe) of knee pain and disability, were recruited for this study. Fifty consecutive patients (31 f. and $19 \mathrm{~m}$.), mean age $52.7 \pm 13.8$ years, with different levels of gonarthrotic pain and disability accepted to participate to this pilot study after being informed of the aims and methods. All participants signed an informed consent form. This study was performed in accordance to the declaration of Helsinki.

\section{II.3. Evaluation of the outcome.}

Assessment of the outcome was done by mean of the Oxford knee pain and disability questionnaire [10]. Scoring was performed at baseline, and at the end of the biophysical delivery after three months, as comparison. according to the following directory:

Scoring 0-19 is a severe joint condition corresponding to a Level 3 of pain and disability.

Scoring 0-29 is a moderate joint condition corresponding to a Level 2 of pain and disability.

Scoring 30-39 is a mild joint condition corresponding to a Level 1 of pain and disability.

Scoring 40-48 is a satisfactory joint condition corresponding to a Level 0 of pain and disability.

\section{II.4. Biophysical therapy procedure.}

A single session, 2-step protocol, of a biophysical procedure was administered to each patient. The 1st step consisted of delivering the program "regulation therapy" settled on the Med Select 729 device (Wegamed, GmbH, Essen, Germany) to record the endogenous input signals at the painful and 
impaired knee region of each patient and of delivering the therapeutic electromagnetic output signals through an electromagnetic, full body, carpet on which the patient laid on for $10 \mathrm{~min}$. The 2 nd step consisted of delivering the program "pain therapy" selected from the touch screen of the Med Select 729 device in order to record the endogenous input signals at the painful knee region and deliver the therapeutic output signals on the same knee pain site for 10 minutes through a couple of magnetic probes. This electro-medical device operates in the low frequency range (carrying frequencies range $0-20 \mathrm{~Hz}$, and modulating frequencies range $0-20 \mathrm{kHz}$ ) delivered through a magnetic field with intensity in the range of the Earth's one, with a maximum of $50 \mu \mathrm{T}$. Along both therapy steps an Electro Magnetic Information Transfer Through Aqueous System procedure [11]-[12]-[13]-[14] was, synchronously, used to record the same pattern of therapeutic signals onto a commercial available aqueous solution (Nomabit Base, Named, Italy) according to previous clinical reports [3]-[4]-[5], [15]-[16]-[17]. The drops were, then, self-administered by the patients at home along a period of three months.

\section{II.5. Statistical Analysis.}

Statistics was performed with Student's t-test using Instat software (GraphPad, San Diego, CA, USA). A p-value $<0.05$ was considered as statistically significant.

\section{RESULTS}

Patients were classified according to their initial score as having a severe (score 0-19) level 3, moderate (score 20-29) level 2, mild (score 30-39) level 1, or satisfactory (score 40-48) level 0, knee condition. A global reduction in the knee pain and disability scoring was observed after 3 months following a single biophysical intervention, in fact mean start level was $2.1 \pm 0.71$, while mean end level was $0.24 \pm 0.52$ $(\mathrm{p}<0.0001)$. Start score was $23.2 \pm 6.9$ while end score was $43.5 \pm 5.1(\mathrm{p}<0.0001)$. Figure one reports the variation of level and scoring at the start and the end of the biophysical treatment.
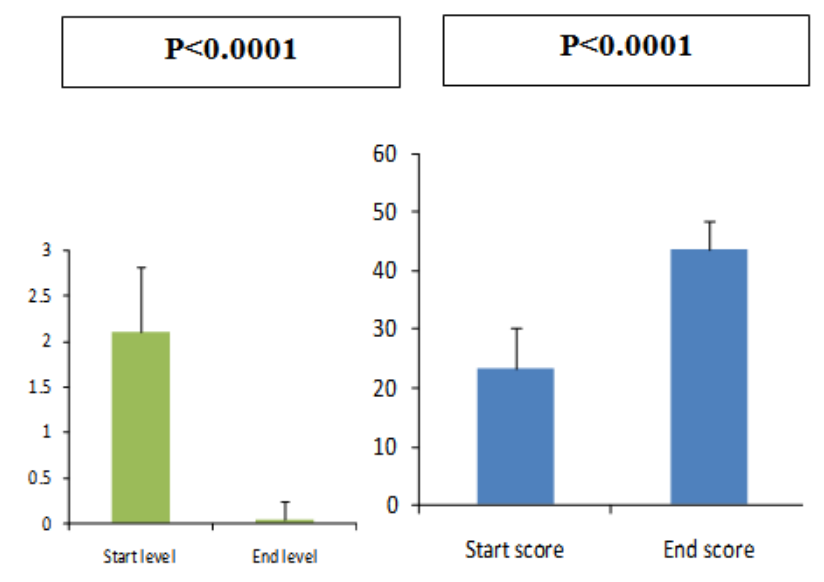

Figure 1. Variation of knee pain and disability level and score from the start to the end.

\section{DISCUSSION}

Our working hypothesis [11] is that the Quantum Electro Dynamic (QED) description of living systems and aqueous systems [14], can account for the mechanism by which an aqueous system could allows to record, store, and transfer to a patient the electromagnetic pattern of its own endogenous signals being able to induce recovery from knee pain and disability. A resonance effect occurring during all the steps of this process is, very likely, responsible for the realization of the therapeutic effect [8] even for a very small intensity of the triggering effect due to non-linearity of human organism [19]. Since the epidemiology of knee pain and disability is becoming more and more relevant [1], due to growing of osteoarthritis incidence, the identification of any safe and effective integrative tool for its management in the primary care setting is emerging in order to both slow down the progression of osteoarthritis, and improve quality of life related to pain and disability. Moreover, biophysical therapies are consistent with the rising quest of a personalized medicine [9], [20] and with the effort to link personal care to population care in general practice [21] in order to develop a true tailored health care [22] allowing to cope with the widespread burden of musculoskeletal diseases [2].

\section{CONCLUSIONS}

Larger studies are certainly warranted to confirm these preliminary report, nevertheless, our study provides an interesting evidence that a biophysical approach to knee pain and disability in a primary care setting can be feasible and effective both reducing the burden of pain, and improving the quality of life related to articular function. Moreover, since the Electro Magnetic Information Transfer Through Aqueous System procedure allows the self-administration of the recorded drops by the patients at home along a period of three months, this biophysical protocol is promising to be both time sparing and cost effective [14].

\section{DECLARATION OF INTEREST}

The authors report no declarations of interest.

\section{REFERENCES}

[1] M. Cross, E. Smith, D. Hoy, S. Nolte, I. Ackerman, M. Fransen, L. Bridgett, S. Williams, F. Guillemin, C.L. Hill, L.L. Laslett, G. Jones, F. Cicuttini, R. Osborne, T. Vos, R. Buchbinder, A. Woolf, and L. March, "The global burden of hip and knee osteoarthritis: estimates from the global burden of disease 2010 study." Ann Rheum Dis., 73(7), 2014: pp. 1323-30

[2] C. Palazzo, J.F. Ravaud, A. Papelard, P. Ravaud, and S. Poiraudeau, "The burden of musculoskeletal conditions." PLoS One., 9(3), 2014: e90633.

[3] A. Foletti, P. Baron, E. Sclauzero, G. Bucci, A. Rinaudo, and R. Rocco, "Assessment of biophysical therapy in the management of pain in current medical practice compared with ibuprofen and placebo: a pilot study." J Biol Regul Homeostat Agents., 28(3), 2014: pp. 431-439.

[4] A. Foletti, and J. Pokorný, "Biophysical approach to low back pain: a pilot report." Electromagn Biol Med., 34(2), 2015: pp. 156-159.

[5] A. Foletti, and P. Baron, "Towards a biophysical approach to different levels of low back pain." Progress in Electromagnetic Research Symposium (PIERS), Shanghai, China, August 8-11 2016, pp. 592-595.

[6] M. Cipollone, and A. Foletti, "Improvement of cervical spine mobility with a biophysical treatment." In Progress in Electromagnetic Research Symposium (PIERS), Shanghai, China, August 8-11 2016, pp. 1404-1407.

[7] A. Foletti, and S. Grimaldi, "Systems Information Therapy and the central role of the brain in allostasis.", J Phys: Conf Ser., 329, 2011: 012027. 


\section{World Journal of Research and Review (WJRR) \\ ISSN:2455-3956, Volume-5, Issue-6, December 2017 Pages 48-50}

[8] A. Foletti, S. Grimaldi, A. Lisi, M. Ledda, and A.R. Liboff, "Bioelectromagnetic medicine: The role of resonance signaling." Electromagn Biol Med., 32(4), 2013: pp. 484-499.

[9] A.M. Henney, "The promise and challenge of personalized medicine: aging populations, complex diseases, and unmet medical need.", Croat Med J., 53(3), 2012: pp. 207-210.

[10] R. Padua, G. Zanoli, E. Ceccarelli, E. Romanini, R. Bondì, and A. Campi A., "The Italian version of the Oxford 12-item Knee Questionnaire-cross-cultural adaptation and validation.” Int Orthop.; 27(4), 2003: pp. 214-6.

[11] A. Foletti, M. Ledda, S. Piccirillo, S. Grimaldi, A. Lisi, "Electromagnetic information delivery as a new tool in translational medicine.” Int J Clin Exp Med., 7(9), 2014: pp. 2550-2556.

[12] A. Foletti, M. Ledda, S. Grimaldi, E. D’Emilia, L. Giuliani, A. Liboff, and A. Lisi, "The trail from quantum electro dynamics to informative medicine.”, Electromagn Biol Med., 34(2), 2015: pp. 147-150.

[13] R.L. Norman, J. Dunning-Davies, J.A. Heredia-Rojas, and A. Foletti A., "Quantum Information Medicine: Bit as It- the future direction of medical science: antimicrobial and other potential nontoxic treatments.", World Journal of Neuroscience., 6 (3), 2016: pp. 193-207.

[14] A. Foletti, M. Ledda, M.G. Lolli, S. Grimaldi, and A. Lisi, "Electromagnetic Information Transfer Through Aqueous System." Electromagn Biol Med., 36(3), 2017: pp. 289-294.

[15] A. Foletti, and M. Cozzolino, "Looking for a biophysical approach to early stages of chronic kidney disease.” In: Proceedings PIERS, Progress in Electromagnetics Research Symposium, Prague, Czech Republic, July 6-9 2015, pp. 171-174.

[16] I. Ferrara, and A. Foletti A., "Steps towards a biophysical approach to refractory gynecological infections.” In: Proceedings PIERS, Progress in Electromagnetics Research Symposium, Prague, Czech Republic, July 6-9 2015, pp. 175-178.

[17] A. Foletti, and P. Baron, "Biophysical approach to minor Anxiety and Depressive Disorders." In Progress in Electromagnetic Research Symposium (PIERS), Shanghai, China, August 8-11 2016, pp. 1400-1403.

[18] A. De Ninno, E. Del Giudice, L. Gamberale, and A. Congiu Castellano, "The structure of liquid water emerging from the vibrational spectroscopy: Interpretation with QED theory." Water., 6, 2014: pp. $13-25$.

[19] L. Brizhik, and A. Foletti, "Nonlinear quantum phenomena and biophysical aspects of complexity related to health and disease.", J Biol Regul Homeostat Agents., 28(3), 2014: pp. 367-376.

[20] M.A. Hamburg, and F.S. Collins, "The path to personalized medicine." N Engl J Med., 363(4), 2010: pp. 301-304.

[21] S. Buetow, L. Getz, P. Adams, "Individualized population care: linking personal care to population care in general practice." J Eval Clin Pract., 14(5), 2008: pp. 761-6.

[22] S. Louca, "Personalized medicine - a tailored health care system: challenges and opportunities.", Croat Med J., 53(3), 2012: 211-213.

\section{Alberto Foletti}

Is a Medical Doctor, specialized in General Surgery with a Master Degree in Neuro Psycho Physical Optimization and CRM Therapy.

$\mathrm{He}$ is research associate at the Institute of Translational Pharmacology of the National Research Council in Rome, Italy.

$\mathrm{He}$ is also the scientific director of the Clinical Biophysics International Research Group, Lugano, Switzerland, aimed to evaluate, independently, the possible clinical applications of biophysical methods as emerging tools in bioelectronic medicine.

Research interests: biophysical signaling in biological systems, role of aqueous systems in biology and medicine, role of coherence and nonlinearity as well as biophysical aspects of complexity in health and disease dynamics, translational aspects and clinical applications of biophysical methods.

$\mathrm{He}$ is author and coauthor of several peer-reviewed papers indexed in the international databases, and he has been lecturer and chairman at international conferences and symposia in the field of biophysics.

\section{Paolo Baron}

Is a Medical Doctor, Pscychoterapist, and work as a General Practitioner in Palmanova, Friuli Venezia Giulia, Italy.

$\mathrm{He}$ is in charge as clinical research activities committee member for General Practitioners.

$\mathrm{He}$ is also an active member of the Clinical Biophysics International Research Group, Lugano, Switzerland, aimed to evaluate, independently, the possible clinical applications of biophysical methods as emerging tools in bioelectronic medicine.

Research interests: evaluation and clinical applications of biophysical methods.

$\mathrm{He}$ is coauthor of some peer-reviewed papers indexed in the international databases, and he has been lecturer at international conferences and symposia in the field of biophysics. 